\title{
Passive Source Localization of Sensor Arrays
}

\author{
Junli Liang and Ding Liu \\ $X i^{\prime}$ an University of Technology, Xi'an, \\ China
}

\section{Introduction}

Passive source localization is a key issue in sensor array signal processing such as sonar, radar, wireless communication, microphone array speech processing, seismology, electronic surveillance and medical imaging, and thus receives significant attention. Although a variety of advanced algorithms, for example MUltiple SIgnal Classification (MUSIC), Estimation of Signal Parameters via Rotational Invariance Techniques (ESPRIT), and Propagator Method (PM), have been developed, there are still some problems: (i)For twodimensional (2D) directions-of-arrival (DOA) estimation, the failure in pairing causes severe performance degradation; (ii) In some practical applications, the signals received by a sensor array may come from multiple near-field sources or multiple far-field sources or their mixture. Due to different signal models for near-field and far-field sources, the existing algorithms cannot deal with them simultaneously well; and (iii) For joint azimuth and elevation direction finding, the existing estimators often encounter an estimation failure problem especially when elevation angles are between 70 and 90 degrees. In this chapter, several high-resolution methods are presented to overcome these difficulties.

In Section 1.2, a novel 2D DOA estimation algorithm without match procedure in the Lshaped array geometry is proposed. It is well known that two matched electric angles (functions of elevation and azimuth angles) must be obtained before elevation and azimuth angles are estimated. However, the failure in pairing would cause severe performance degradation. By introducing a novel electric angle, the L-shaped array configuration without any rotational invariance property between two orthogonal uniform linear sub-arrays evolves into some particular rotational invariance geometry. Thus, the steering vector is separated into two parts. One can be estimated by the rank-reduction ESPRIT algorithm and the other is obtained from the eigenvalue decomposition of one particular matrix. Finally, the elevation and azimuth angles can be easily obtained from the recovered steering vector to avoid pairing. Although it is developed for the L-shaped array configuration, the proposed algorithm can be easily extended to other array geometries such as two parallel linear sub-arrays, the rectangular array, and the symmetric circular array. In addition, the method can be used to form the rank-reduction propagator method. electric angle

In Section 1.3, a common signal model for "any-field" sources (i.e., near-field sources or farfield sources or their mixture) is given and a two-stage MUSIC algorithm is developed to localize "any-field" sources. In the first stage, one special cumulant matrix is derived and the related virtual "steering vector" is the function of the common electric angle in both 
near-field and far-field signal models so that DOA of near-field or far-field can be obtained from this electric angle using the conventional high-resolution MUSIC algorithm. In the second stage, another particular cumulant matrix is derived, in which the virtual "steering matrix" has full column rank no matter whether the received signals are multiple near-field sources or multiple far-field ones or their mixture. More importantly, the virtual "steering vector" can be separated into two parts, in which the first one is the function of the common electric angle in both signal models, whereas the second part is the function of the electric angle that exists only in the near-field signal model. Furthermore, by substituting the common electric angle, which is estimated in the first stage into one special Hermitian matrix formed from another MUSIC spectral function, the range of near-field sources can be obtained from the eigenvector of the Hermitian matrix. Although it is developed for azimuth angle (and range) estimation only, it can be developed further for the joint azimuth and elevation angles (as well as range) estimation.

In Section 1.4, a novel high-accuracy estimator for elevation angle is developed to avoid the estimation failure problem encountered in the conventional elevation estimators. Firstly, three cumulant matrices are constructed using fourth-order cumulants of some properly chosen array outputs of a specially designed volume array to increase the array aperture. Secondly, a parallel factor (PARAFAC) model of cumulant matrices in the cumulant domain is formed to avoid pairing parameters. Finally, a flexible and high-resolution elevation angle estimator is derived from multiple electric angles, which are solved from the above steps.

\section{2D DOA estimation without match procedure}

Estimation of 2-D DOA is a key issue in sensor array signal processing such as radar, sonar, radio astronomy, and mobile communication systems [1-4]. Similar to other array geometries such as the parallel uniform linear array, the rectangular array and the circular array, there is an un-avoidable parameter association problem in the L-shaped array configuration because the failure in pairing would cause severe performance degradation. This section will give a novel 2-D DOA estimation algorithm, which does not require match procedure.

\subsection{Description of the proposed algorithm}

Let's consider an L-shaped sensor array with $2 M+1$ omni-directional sensors, as shown in Fig. 1. The element placed at the origin is set for the referencing point. The array in the $x-z$ plane consists of two uniform linear sub-arrays with element spacing $d$, each being composed of $M$ elements. Assume that $L$ far-field, no-coherent, narrowband sources impinging on this antenna array. Let $\alpha_{l}$ and $\beta_{l}$ be the elevation and azimuth angles of the $l$-th source, and thus the wave vector $\mathbf{k}_{l}$ containing DOA information can be defined as $\mathbf{\kappa}_{l}=\left[\sin \alpha_{l} \cos \beta_{l}, \sin \alpha_{l} \sin \beta_{l}, \cos \alpha_{l}\right], l=1, \cdots, L$. After being sampled, the signals received by the sensor array can be expressed as

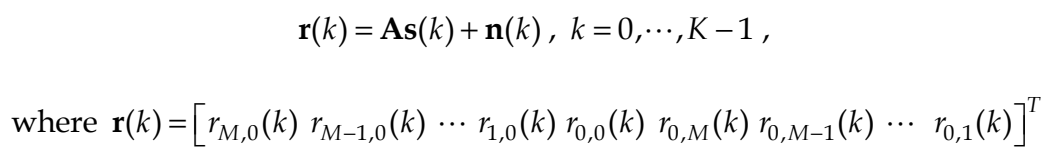




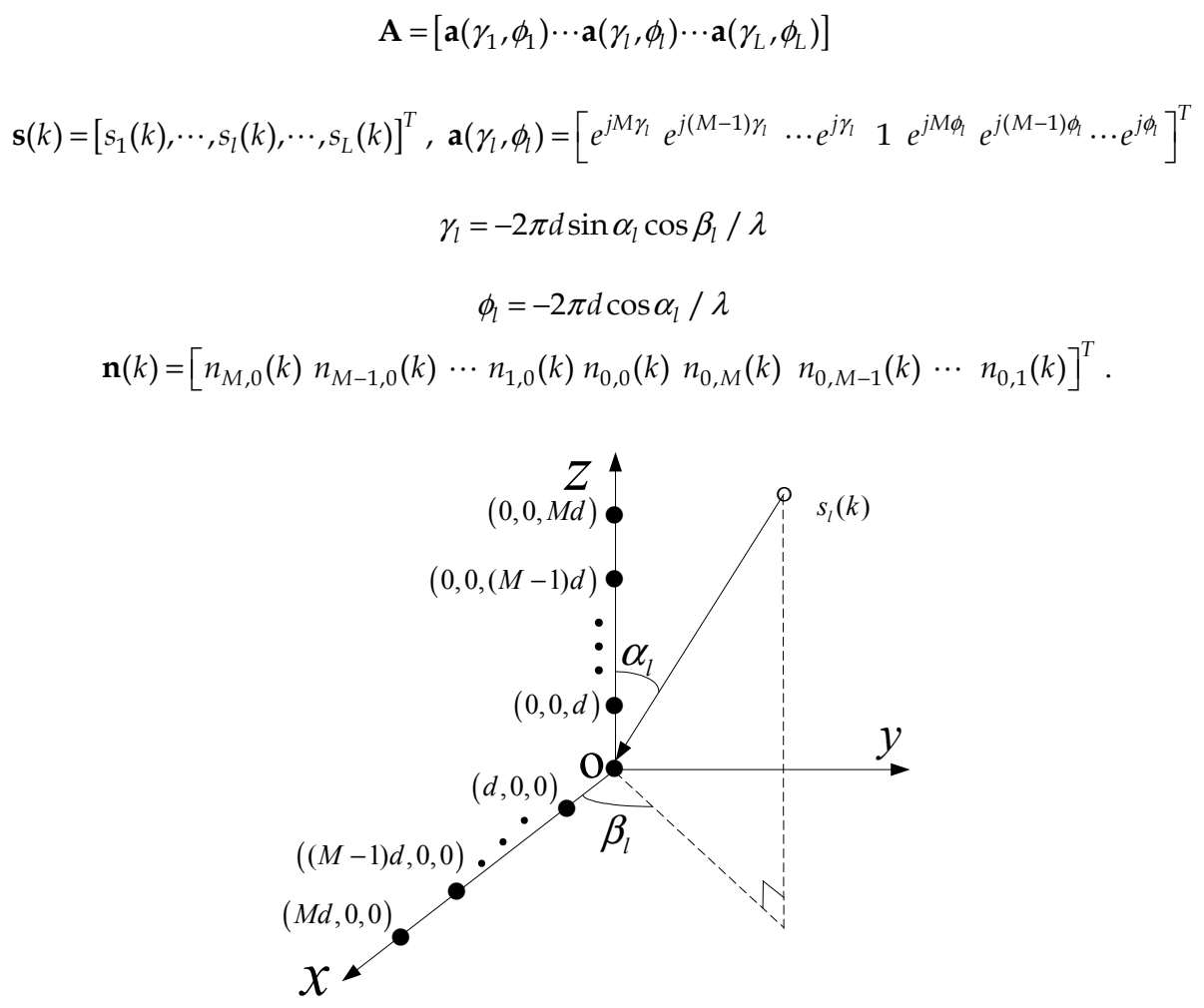

Fig. 1. L-shaped sensor array configuration

The auto-correlation matrix of $\mathbf{r}(k)$ can be expressed as $\mathbf{R}=E\left[\mathbf{r}(k) \mathbf{r}^{H}(k)\right]$, $=\mathbf{A R}_{s} \mathbf{A}^{H}+\sigma_{n}^{2} \mathbf{I}_{2 M+1}$, where $\mathbf{R}_{s}=E\left[\mathbf{s}(k) \mathbf{s}^{H}(k)\right]$, and its eigen-value decomposition (EVD) yields

$$
\mathbf{R}=\mathbf{U V U} \mathbf{U}^{H}=\mathbf{U}_{s} \mathbf{V}_{s} \mathbf{U}_{s}^{H}+\mathbf{U}_{n} \mathbf{V}_{n} \mathbf{U}_{n}^{H}=\left[\mathbf{u}_{1}, \cdots, \mathbf{u}_{2 M+1}\right] \operatorname{diag}\left[v_{1}, \cdots, v_{2 M+1}\right]\left[\mathbf{u}_{1}, \cdots, \mathbf{u}_{2 M+1}\right]^{H},
$$

where $\mathbf{V}$ is the diagonal matrix with the eigen-values arranged as $v_{1} \geq \cdots \geq v_{L}>v_{L+1} \geq \cdots \geq v_{2 M+1}$, the diagonal matrix $\mathbf{V}_{n} \in R^{(2 M+1-L) \times(2 M+1-L)}$ is composed of eigen-values $v_{L+1}, v_{L+2}, \cdots, v_{2 M+1} ; \mathbf{U}_{n} \in C^{(2 M+1) \times(2 M+1-L)}$ consists of the eigenvectors related to $v_{L+1}, v_{L+2}, \cdots, v_{2 M+1}$, spanning the noise subspace of $\mathbf{R}$.

Let's define $e^{j \theta_{l}}=e^{j\left(\phi_{l}-\gamma_{l}\right)}$, and thus the steering vector be written in another form as:

$$
\mathbf{a}\left(\gamma_{l}, \phi_{l}\right)=\left[\begin{array}{llllll}
e^{j M \gamma_{l}} & e^{j(M-1) \gamma_{l}} & \cdots e^{j \gamma_{l}} & 1 & e^{j M \theta_{l}} \times e^{j M \gamma_{l}} & e^{j(M-1) \theta_{l}} \times e^{j(M-1) \gamma_{l}} \cdots e^{j \theta_{l}} \times e^{j \gamma_{l}}
\end{array}\right]^{T}
$$

Furthermore, $\mathbf{a}(\gamma, \phi)$ can be separated into two parts, i.e. $\mathbf{a}(\gamma, \phi)=\mathbf{a}_{1}\left(e^{j \theta}\right) \mathbf{a}_{2}\left(e^{j \gamma}\right)$, where $(M+1)$-dimensional vector $\mathbf{a}_{2}\left(e^{j \gamma}\right)=\left[e^{j M \gamma} e^{j(M-1) \gamma} \cdots e^{j \gamma} 1\right]^{T}$ and $(2 M+1) \times(M+1)$ - dimensional matrix 


$$
\mathbf{a}_{1}\left(e^{j \theta}\right)=\left[\begin{array}{c}
\mathbf{I}_{(M+1) \times(M+1)} \\
\operatorname{diag}\left\{e^{j M \theta}, \cdots, e^{j \theta}\right\} \\
\mathbf{0}_{M \times 1}
\end{array}\right]
$$

Based on the theory that the noise subspace $\mathbf{U}_{n}$ is orthogonal to the range space of $\mathbf{A}$, $\mathbf{U}_{n}^{H} \mathbf{a}\left(\gamma_{l}, \phi_{l}\right)=\mathbf{0}, l=1, \cdots, L$, the electric angle pair $\left\{\gamma_{l}, \phi_{l}\right\}, l=1, \cdots, L$ can be found from the $L$ deepest minima of the following MUSIC spectral function:

$$
\begin{aligned}
& f_{1}(\gamma, \phi)=\mathbf{a}^{H}\left(\gamma_{l}, \phi_{l}\right) \mathbf{U}_{n} \mathbf{U}_{n}^{H} \mathbf{a}\left(\gamma_{l}, \phi_{l}\right)=\mathbf{a}_{2}^{H}\left(e^{j \gamma_{l}}\right) \mathbf{a}_{1}^{H}\left(e^{j \theta_{l}}\right) \mathbf{U}_{n} \mathbf{U}_{n}^{H} \mathbf{a}_{1}\left(e^{j \theta_{l}}\right) \mathbf{a}_{2}\left(e^{j \gamma_{l}}\right) \\
& =\mathbf{a}_{2}^{H}\left(e^{j \gamma_{l}}\right) \mathbf{C}\left(e^{j \theta_{l}}\right) \mathbf{a}_{2}\left(e^{j \gamma_{l}}\right)=0
\end{aligned}
$$

where

$$
\mathbf{C}\left(e^{j \theta}\right)=\mathbf{a}_{1}^{H}\left(e^{j \theta}\right) \mathbf{U}_{n} \mathbf{U}_{n}^{H} \mathbf{a}_{1}\left(e^{j \theta}\right)
$$

is an $(M+1) \times(M+1)$-dimensional Hermitian matrix.

Note that $\mathbf{a}_{1}\left(e^{j \theta_{l}}\right) \neq \mathbf{0}$ and $\mathbf{a}_{2}^{H}\left(e^{j \gamma_{l}}\right) \mathbf{C}\left(e^{j \theta_{l}}\right) \mathbf{a}_{2}\left(e^{j \gamma_{l}}\right)=0, l=1,2, \cdots, L$. From Eq. (5)-(6), it can be seen that if and only if $e^{j \theta}=e^{j \theta_{l}}$, the matrix $\mathrm{C}\left(e^{j \theta}\right)$ drops rank, or equivalently, when the polynomial of $x=e^{j \theta}, f_{2}(x)=\operatorname{det}\{\mathbf{C}(x)\}=0$. Obviously, $\hat{x}$, lying inside the unit circle and being closest to the unit circle, is actually the signal root.

Eq. (5) implies that by substituting the estimated $e^{j \hat{\theta}_{l}}$ into $\mathbf{C}\left(e^{j \theta}\right)$ in Eq. (5), $\hat{\gamma}_{l}$ can be found from the minima of the following function:

$$
\hat{\gamma}_{l}=\min _{\gamma} \mathbf{a}_{2}^{H}\left(e^{j \gamma}\right) \mathbf{C}\left(e^{j \hat{\theta}_{l}}\right) \mathbf{a}_{2}\left(e^{j \gamma}\right)
$$

the minima of which indicates estimation.

When $\phi_{p}-\gamma_{p} \neq \phi_{q}-\gamma_{q}+2 h \pi, h \in\{-1,0,1\}, p, q \in\{1, \cdots, L\}$, i.e., $\theta_{p} \neq \theta_{q}$, Eq. (7) implies that $\mathbf{a}_{2}\left(e^{j \hat{\gamma}_{p}}\right)$ is just the unique eigenvector corresponding to the smallest eigen-value of $\mathbf{C}\left(e^{j \hat{\theta}_{p}}\right)$. However, when $\phi_{p}-\gamma_{p}=\phi_{q}-\gamma_{q}+2 h \pi, \mathbf{a}_{2}\left(e^{j \hat{\gamma_{p}}}\right)$ is no longer the unique eigenvector corresponding to the smallest eigen-value of $\mathbf{C}\left(e^{j \hat{\theta}_{p}}\right)$. The eigen-value decomposition (EVD) of $\mathbf{C}\left(e^{j \hat{\theta}_{p}}\right)$ yields $\mathbf{C}\left(e^{j \hat{\theta}_{p}}\right)=\left[\tilde{\mathbf{u}}_{1}, \cdots, \tilde{\mathbf{u}}_{M+1}\right] \operatorname{diag}\left[\tilde{v}_{1}, \cdots, \tilde{v}_{M+1}\right]\left[\tilde{\mathbf{u}}_{1}, \cdots, \tilde{\mathbf{u}}_{M+1}\right]^{-1}$, where the eigen-values are arranged as $\left|v_{1}\right| \geq\left|v_{2}\right| \geq \cdots>\left|v_{M}\right|=\left|v_{M+1}\right|$. It is obvious that under the case $\phi_{p}-\gamma_{p}=\phi_{q}-\gamma_{q}+2 h \pi \quad \mathbf{a}_{2}\left(e^{j \hat{\gamma}_{p}}\right)$ and $\mathbf{a}_{2}\left(e^{j \hat{\gamma}_{q}}\right)$ are the linear combinations of two eigenvectors $\left\{\tilde{\mathbf{u}}_{M}, \tilde{\mathbf{u}}_{M+1}\right\}$, which are orthogonal to $\tilde{\mathbf{U}}_{\mathrm{s}}=\left\{\tilde{\mathbf{u}}_{1}, \tilde{\mathbf{u}}_{2}, \cdots, \tilde{\mathbf{u}}_{M-1}\right\}$. Obviously, both $e^{j \hat{\gamma}_{p}}$ and $e^{j \hat{\gamma}_{q}}$ are the roots of $f_{3}(x)=\mathbf{a}_{2}^{H}(x) \tilde{\mathbf{U}}_{s} \tilde{\mathbf{U}}_{s}^{H} \mathbf{a}_{2}(x)=0$.

From the estimates $\left\{e^{j \hat{\gamma}_{l}}, e^{j \hat{\theta}_{l}}\right\}$, the elevation and azimuth angle estimates can be given as $\hat{\alpha}_{l}=\arccos \left(-\angle\left(e^{j \hat{\theta}_{l}} \times e^{j \hat{\gamma}_{l}}\right)^{\prime} \lambda /(2 \pi d)\right)$ and $\hat{\beta}_{l}=\arccos \left(-\left(\angle e^{j \hat{\gamma}_{l}}\right) \lambda /\left(2 \pi d \sin \hat{\alpha}_{l}\right)\right)$, respectively. Since $\mathbf{a}_{2}\left(e^{j \hat{\gamma}_{l}}\right)$ is related to $\mathbf{C}\left(e^{j \hat{\theta}_{l}}\right)$ (i.e., corresponding to $\mathbf{a}_{1}\left(e^{j \hat{\theta}_{l}}\right)$ ), the proposed algorithm can avoid pairing parameters. In addition, it avoids the spectral search because both $e^{j \hat{\gamma}_{l}}$ and $e^{j \hat{\theta}_{l}}$ are estimated by solving polynomial roots. 


\subsection{Simulation results}

To verify the effectiveness of the proposed algorithm, let's consider an L-shaped array with 13 elements as shown in Fig.1. These sensor locations are in unit of $d=\lambda / 2$. Two uncorrelated equivalent-power sources $\left(e^{j 0.2 \pi k}\right.$ and $\left.e^{j 0.25 \pi k}\right)$, respectively with DOAs of $\left(\alpha_{1}=60^{\circ}, \beta_{1}=35^{\circ}\right)$ and $\left(\alpha_{2}=40^{\circ}, \beta_{2}=55^{\circ}\right)$, impinge on this array. The root mean square error (RMSE) is used as the performance measure. All results provided are based on 500 independent runs. The RMSE for DOA estimation is defined as

$$
\operatorname{RMSE}(\text { the } l \text { th signal })=\sqrt{\frac{1}{500} \sum_{i=1}^{500}\left(\hat{\alpha}_{i, l}-\alpha_{l}\right)^{2}}
$$

in which $\hat{\alpha}_{i, l}$ (in unit of degree) stands for the estimation of the $l$-th elevation $\alpha_{l}$ in the $i$ th trial. For comparison, the propagator method $[6,9]$ and the ESPRIT method $[8,11]$ with correct pairing are simultaneously executed.

In the first experiment, the effect of signal-to-noise (SNR) on the performance of the proposed algorithm is investigated. The number of snapshots is set to 400 and the SNR varies from 0 to $30 \mathrm{~dB}$. The averaged performances (RMSE of elevation and azimuth angle estimations versus SNR for two sources) over 500 Monte Carlo runs are shown in Figs. 2 and 3. As expected, when the SNR increases, the RMSE of the estimated parameters decrease. In addition, it is observed that the proposed algorithm improves the performance slightly compared to the conventional ESPRIT algorithm, which must have a precise association procedure.

In the second experiment, the influence of snapshot number on the performance of the proposed algorithm is explored. The same parameters as that of the second experiment are used, except that the SNR is fixed at $10 \mathrm{~dB}$ and the number of snapshots varies from 200 to 2000. The averaged performances (RMSE of elevation and azimuth angle estimations versus snapshot number for two sources) over 500 Monte Carlo runs are shown in Figs. 4 and 5. From these figures, it can be seen that RMSE of the elevation and azimuth estimations decrease as snapshot number increases. In addition, the proposed algorithm has higher estimation accuracy than the ESPRIT method.

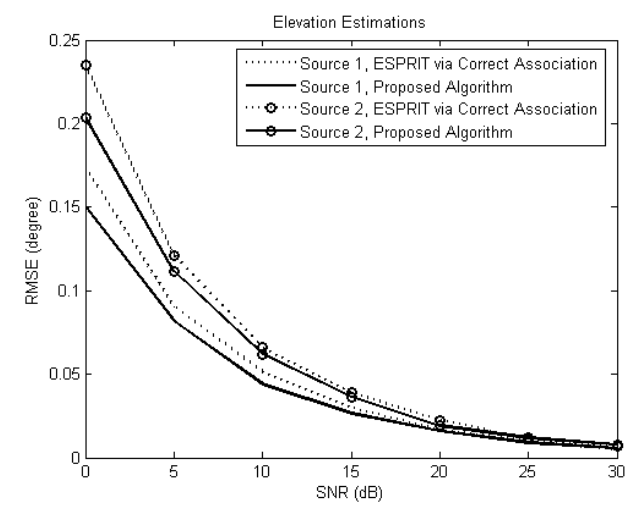

Fig. 2. RMSE of elevation angle estimations versus SNR 


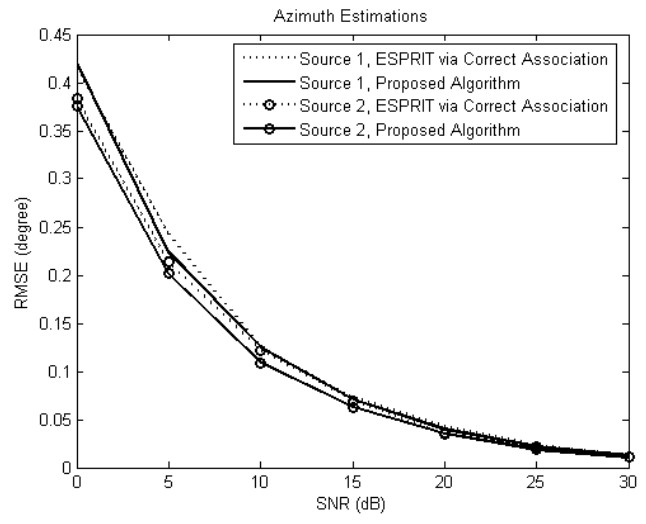

Fig. 3. RMSE of azimuth angle estimations versus SNR

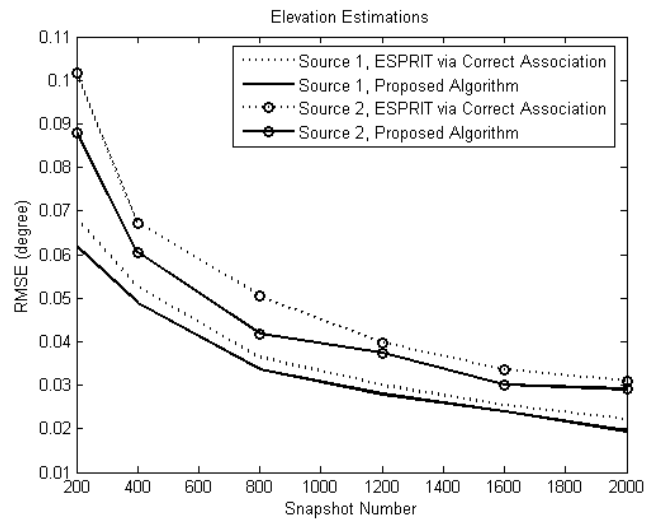

Fig. 4. RMSE of elevation angle estimations versus snapshot number

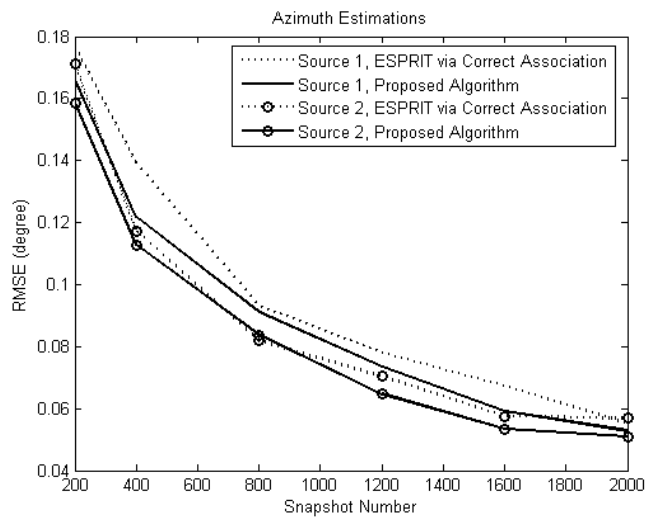

Fig. 5. RMSE of azimuth angle estimations versus snapshot number 
From the above experiments, it can be seen that (i) since $\mathbf{a}_{2}\left(e^{j \hat{\gamma}_{l}}\right)$ is related to $\mathbf{C}\left(e^{j \hat{\theta}_{l}}\right)$ (i.e. corresponding to $\mathbf{a}_{1}\left(e^{j \hat{\theta}_{l}}\right)$ ), the proposed algorithm can avoid pairing parameters; and (ii) the proposed algorithm avoids the spectral search due to that both $e^{j \hat{\gamma}_{l}}$ and $e^{j \hat{\theta}_{l}}$ are estimated by solving polynomial roots.

\section{Passive localization of mixed near-field and far-field sources}

In some practical applications, the signals received by an array are often the mixture of nearfield and far-field sources, such as speaker localization using microphone arrays and guidance (homing) systems [12-19]. For example, in the application of speaker localization using microphone arrays, each speaker may be in the near-field or far-field of the array [16]. In this case, either existing near-field source localization methods or far-field source those may fail in localizing mixed near-field and far-field sources. This section will give a new passive source localization algorithm, which can localize near-field sources or far-field sources or their mixture.

\subsection{Description of the proposed algorithm}

Consider that $L$ (near-field ${ }^{1}$ or far-field) narrowband, independent radiating sources, impinge on the uniform linear array (ULA) with $2 N+1$ elements as shown in Fig.6. Let the 0 th sensor be the phase reference point. After sampled with a proper rate that satisfies the Nyquist rate, the signal received by the $i$ th sensor can be expressed as [5-11]

$$
x_{i}(k)=\sum_{l=1}^{L} s_{l}(k) e^{j \tau_{i l}}+n_{i}(k),-N \leq i \leq N, k=0, \cdots, K-1,
$$

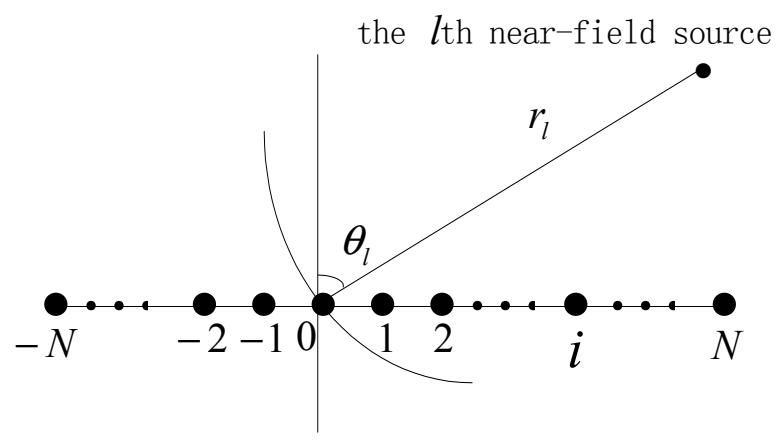

Fig. 6. Uniform linear array configuration

\footnotetext{
${ }^{1}$ Note that Fresnel zone (i.e. near-field) lies in the radiating zone $\left[\frac{1}{2 \pi} \lambda, \frac{1}{\lambda} 2 D^{2}\right]$, where $\lambda$ and $D$ are signal wavelength and array dimension, respectively (see [4] for details). Whereas far-field means the radiating zone beyond $\left[0, \frac{1}{\lambda} 2 D^{2}\right]$.
} 
Where $K$ is the snapshot number, $s_{l}(k)$ is the $l$ th narrowband source, $n_{i}(k)$ is the additive Gaussian noise. In addition, $\tau_{i l}$ is the delay associated with the $l$ th source propagation time between the 0 th and $i$ th sensors. If the $l$ th source is near-field one (the azimuth DOA $\theta_{l}$ and the range $\left.r_{l}\right), \tau_{i l}=i \gamma_{l}+i^{2} \phi_{l}$, where $\gamma_{l}$ and $\phi_{l}$ are called electric and given by

$$
\gamma_{l}=-2 \pi \frac{d}{\lambda} \sin \left(\theta_{l}\right), \text { and } \phi_{l}=\pi \frac{d^{2}}{\lambda r_{l}} \cos ^{2}\left(\theta_{l}\right) .
$$

Otherwise, if the $l$ th source is far-field one, $\tau_{i l}=i \gamma_{l}[2,3]$, where $\phi_{l}$ is approximated by zero due to father range of far-field source. Therefore, a far-field source can be considered as the special near-field one, where $\phi_{l}=0$.

In a matrix form, Eq. (1) can be written as

$$
\mathbf{x}(k)=\mathbf{A s}(k)+\mathbf{n}(k)
$$

$$
\begin{gathered}
\text { where } \mathbf{A}=\left[\mathbf{a}\left(\gamma_{1}, \phi_{1}\right) \cdots \mathbf{a}\left(\gamma_{l}, \phi_{l}\right) \cdots \mathbf{a}\left(\gamma_{L}, \phi_{L}\right)\right] \\
\mathbf{n}(k)=\left[n_{-N}(k), \cdots, n_{0}(k), n_{1}(k), \cdots, n_{N}(k)\right]^{T}, \\
\mathbf{s}(k)=\left[s_{1}(k), \cdots, s_{l}(k), \cdots, s_{L}(k)\right]^{T} \\
\mathbf{x}(k)=\left[x_{-N}(k), \cdots, x_{0}(k), x_{1}(k), \cdots, x_{N}(k)\right]^{T} .
\end{gathered}
$$

Note that the form of steering vector $\mathbf{a}\left(\gamma_{l}, \phi_{l}\right)$ depends on whether the $l$ th source is far-field one or near-field one. If this source is near-field one,

$$
\mathbf{a}\left(\gamma_{l}, \phi_{l}\right)=\left[e^{j\left[(-N) \gamma_{l}+(-N)^{2} \phi_{l}\right]} e^{j\left[(-N+1) \gamma_{l}+(-N+1)^{2} \phi_{l}\right]} \cdots e^{j\left[N \gamma_{l}+N^{2} \phi_{l}\right]}\right]^{T} .
$$

Otherwise, if this source lies in the far field,

$$
\mathbf{a}\left(\gamma_{l}, \phi_{l}\right)=\left[e^{j\left[(-N) \gamma_{l}\right]} e^{j\left[(-N+1) \gamma_{l}\right]} \cdots e^{j\left[N \gamma_{l}\right]}\right]^{T} .
$$

Let's begin with the fourth-order cumulant of the sensor outputs, which can be expressed as

$$
\begin{aligned}
& \operatorname{cum}\left\{x_{m}(k), x_{n}^{*}(k), x_{p}^{*}(k), x_{q}(k)\right\} \\
= & \operatorname{cum}\left\{\sum_{l=1}^{L} s_{l}(k) e^{j\left(m \gamma_{l}+m^{2} \phi_{l}\right)},\left(\sum_{l=1}^{L} s_{l}(k) e^{j\left(n \gamma_{l}+n^{2} \phi_{l}\right)}\right)^{*},\left(\sum_{l=1}^{L} s_{l}(k) e^{j\left(p \gamma_{l}+p^{2} \phi_{l}\right)}\right)^{*}, \sum_{l=1}^{L} s_{l}(k) e^{j\left(q \gamma_{l}+q^{2} \phi_{l}\right)}\right\} \\
= & \sum_{l=1}^{L} e^{j\left\{[(m-n)-(p-q)] \gamma_{l}+\left[\left(m^{2}-n^{2}\right)-\left(p^{2}-q^{2}\right)\right] \phi_{l}\right\}} \operatorname{cum}\left\{s_{l}(t), s_{l}^{*}(t), s_{l}^{*}(t), s_{l}(t)\right\} \\
= & \sum_{l=1}^{L} c_{4, s l} e^{j\left[[(m-n)-(p-q)] \gamma_{l}+\left[\left(m^{2}-n^{2}\right)-\left(p^{2}-q^{2}\right)\right] \phi_{l}\right\}}
\end{aligned}
$$

$$
m, n, p, q \in[-N, N]
$$


where $c_{4, s l}=\operatorname{cum}\left\{s_{l}(t), s_{l}^{*}(t), s_{l}^{*}(t), s_{l}(t)\right\}$ is the kurtosis of the $l$ th signal, and the superscript * denotes the complex conjugate.

To construct a matrix with full rank for arbitrary-field sources, let $n=-m$ and $q=0$. Thus, Eq. (11) becomes

$$
\operatorname{cum}\left\{x_{m}(k), x_{-m}^{*}(k), x_{p}^{*}(k), x_{q}(k)\right\}=\sum_{l=1}^{L} c_{4, s l} e^{j 2 m \gamma_{l}}\left(e^{j\left(p \gamma_{l}+p^{2} \phi_{l}\right)}\right)^{*}, \quad m, p \in[-N, N]
$$

Let $\bar{m}=m+N+1$ and $\bar{p}=p+N+1$, and thus $\bar{m}, \bar{p} \in[1,2 N+1]$. Based on the idea from (11)(12), a special $(2 N+1) \times(2 N+1)$-dimensional cumulant matrix $\mathbf{C}$ can be defined, the $(\bar{m}, \bar{p})$ th element of which can be given by

$$
\begin{aligned}
& \mathbf{C}(\bar{m}, \bar{p})=\operatorname{cum}\left\{x_{\bar{m}-N-1}(k), x_{-\bar{m}+N+1}^{*}(k), x_{\bar{p}-N-1}^{*}(k), x_{0}(k)\right\} \\
& =\sum_{l=1}^{L} c_{4, s l} e^{j 2(\bar{m}-N-1) \gamma_{l}}\left(e^{j(\bar{p}-N-1) \gamma_{l}+j(\bar{p}-N-1)^{2} \phi_{l}}\right)^{*} \quad \bar{m}, \bar{p} \in[1,2 N+1]
\end{aligned}
$$

Note that the $(2 N+1) \times(2 N+1)$ matrix $\mathbf{C}$ can be represented in a compact matrix form as $\mathbf{C}=\mathbf{B C}_{4 s} \mathbf{A}^{H}$, where the superscript $H$ stands for the Hermitian transpose, $\mathbf{C}_{4 s}=\operatorname{diag}\left[c_{4, s 1}, \cdots, c_{4, s l}, \cdots, c_{4, s L}\right]$, virtual "steering matrix" $\mathbf{B}=\left[\mathbf{b}\left(\gamma_{1}\right), \cdots, \mathbf{b}\left(\gamma_{l}\right), \cdots, \mathbf{b}\left(\gamma_{L}\right)\right]$, and virtual "steering vector" $\mathbf{b}\left(\gamma_{l}\right)=\left[e^{-j 2 N \gamma_{l}}, e^{-j(2 N-2) \gamma_{l}}, \cdots 1, \cdots, e^{j 2 N \gamma_{l}}\right]^{T}, l=1, \cdots, L$.

The singular value decomposition (SVD) of $\mathbf{C}$ yields

$$
\mathbf{C}=\mathbf{B C}_{4 s} \mathbf{A}^{H}=\mathbf{W} \boldsymbol{\Sigma} \mathbf{Z}^{H}=\left[\mathbf{w}_{1}, \cdots, \mathbf{w}_{2 N+1}\right] \operatorname{diag}\left(\sigma_{1}, \cdots, \sigma_{2 N+1}\right)\left[\mathbf{z}_{1}, \cdots, \mathbf{z}_{2 N+1}\right]^{H}
$$

where $\Sigma$ is the diagonal matrix with the singular values arranged as $\left|\sigma_{1}\right| \geq \cdots \geq\left|\sigma_{L}\right|>\left|\sigma_{L+1}\right| \geq \cdots \geq\left|\sigma_{2 N+1}\right|$. Let $\mathbf{W}_{s} \in C^{(2 N+1) \times L}$, which spans the signal subspace of $\mathbf{B}$, consists of the left singular vectors $\mathbf{w}_{1}, \mathbf{w}_{2}, \cdots, \mathbf{w}_{L}$. Similarly, $\mathbf{Z}_{n} \in C^{(2 N+1) \times(2 N+1-L)}$, which is orthogonal to $\mathbf{A}$, consists of the right singular vectors $\mathbf{z}_{L+1}, \mathbf{z}_{L+2}, \cdots, \mathbf{z}_{2 N+1}$.

Based on the first $2 N$ lines $\mathbf{W}_{s 1}$ and last $2 N$ lines $\mathbf{W}_{s 2}, \gamma_{l}, l=1, \cdots, L$ can be easily estimated from the eigen-values of the following matrix [3] :

$$
\gamma_{l}=\angle(\Phi(l, l)) / 2
$$

where $\boldsymbol{\Phi}=\operatorname{diag}\left[e^{j 2 \gamma_{1}}, \cdots, e^{j 2 \gamma_{l}}, \cdots, e^{j 2 \gamma_{L}}\right]$ is the eigen-value matrix $\mathbf{W}_{s 1}^{\#} \mathbf{W}_{s 2}$, i.e. $\mathbf{W}_{s 1}^{\#} \mathbf{W}_{s 2}=\mathbf{T} \boldsymbol{\Phi} \mathbf{T}^{-1}$.

By substituting the estimate $\hat{\gamma}_{l}$ into $\mathbf{a}(\gamma, \phi)$, the minima of the following function can be found.

$$
\begin{gathered}
\hat{\phi}_{l}=\min _{\phi} \mathbf{a}^{H}\left(\hat{\gamma}_{l}, \phi\right) \mathbf{Z}_{n} \mathbf{Z}_{n}^{H} \mathbf{a}\left(\hat{\gamma}_{l}, \phi\right)=\min _{\phi} \mathbf{a}_{2}^{H}(\phi) \mathbf{a}_{1}^{H}\left(\hat{\gamma}_{l}\right) \mathbf{Z}_{n} \mathbf{Z}_{n}^{H} \mathbf{a}_{1}\left(\hat{\gamma}_{l}\right) \mathbf{a}_{2}(\phi), l=1, \cdots, L \\
\text { where } \mathbf{a}_{1}(\gamma)=\left[\begin{array}{c}
\operatorname{diag}\left\{e^{-j N \gamma}, \cdots, e^{-j \gamma}, 1\right\} \\
\operatorname{diag}^{\text {anti }}\left\{e^{j \gamma}, \cdots, e^{j N \gamma}\right\} \\
\mathbf{0}_{\mathrm{N} \times 1}
\end{array}\right], \quad \mathbf{a}_{2}(\phi)=\left[e^{j N^{2} \phi} e^{j(N-1)^{2} \phi} \cdots e^{j \phi} 1\right]^{T} .
\end{gathered}
$$


Eq. (16) implies that $\mathbf{a}_{2}\left(\hat{\phi}_{l}\right)$ is just the eigenvector corresponding to the smallest eigen-value of $\mathbf{a}_{1}^{H}\left(\hat{\gamma}_{l}\right) \mathbf{Z}_{n} \mathbf{Z}_{n}^{H} \mathbf{a}_{1}\left(\hat{\gamma}_{l}\right)$, and $\hat{\phi}_{l}$ is easily solved from $\mathbf{a}_{2}\left(\hat{\phi}_{l}\right)$.

The DOA and range estimates of the $l$ th source can be in turn expressed as:

$$
\hat{\theta}_{l}=\arcsin \left(-\frac{\hat{\gamma}_{l} \lambda}{2 \pi d}\right)
$$

and

$$
\hat{r}_{l}=\frac{\pi d^{2}}{\lambda \hat{\phi}_{l}} \cos ^{2}\left(\hat{\theta}_{l}\right), l=1, \cdots, L .
$$

In fact, if the $l$ th source is far-field one, the estimate $\hat{\phi}_{l}$ would approach to zero. Thus, whether the $l$ th source is near-field or far-field one can be determined. Since both $\mathbf{B}$ and $\mathbf{A}$ are of full column rank no matter whether the received signals be pure far-field sources or pure near-field sources or mixed far-field and near-field sources, the proposed algorithm can deal with arbitrary-field sources well.

\subsection{Description of the proposed algorithm}

Some simulations are conducted in this section to assess the ability of the proposed algorithm to localize near-field, far-field, as well as mixed near-field and far-field sources.

Two near-field sources are located at $\left\{\theta_{1}=10^{\circ}, r_{1}=0.5 \lambda\right\}$ (i.e. $\left\{\gamma_{1}=-0.2728, \phi_{1}=0.3809\right\}$ ) and $\left\{\theta_{2}=20^{\circ}, r_{2}=1.0 \lambda\right\}$ (i.e. $\left\{\gamma_{2}=-0.5372, \phi_{2}=0.1734\right\}$ ), respectively. The snapshot number and SNR are fixed at 400 and $10 \mathrm{~dB}$. The scatter plot of estimated $\left(\hat{\gamma}_{l}, \hat{\phi}_{l}\right)$ pairs from 500 independent trials using the proposed algorithm, the near-field source localization algorithm (i.e. ESPRIT), and the far-field source localization algorithm (i.e. MUSIC) are shown in Figs. 7-9, respectively. From these figures, it can be seen that the far-field source localization algorithm fails in localizing near-field sources.

The near-field source is located at $\left\{\theta_{1}=10^{\circ}, r_{1}=0.5 \lambda\right\}$ (i.e. $\left\{\gamma_{1}=-0.2728, \phi_{1}=0.3809\right\}$ ); whereas the far-field source is localized at $\left\{\theta_{2}=20^{\circ}, r_{2}=+\infty\right\}$ (i.e. $\left\{\gamma_{2}=-0.5372, \phi_{2}=0\right\}$ ). The snapshot number and SNR are fixed at 400 and $10 \mathrm{~dB}$, respectively. The scatter plot of estimated $\left(\hat{\gamma}_{l}, \hat{\phi}_{l}\right)$ pairs from 500 independent trials using the proposed algorithm, the near-field source localization algorithm (ESPRIT), and the far-field source localization algorithm (MUSIC) are shown in Figs. 10-12, respectively. These figures show that the far-field source localization algorithm (MUSIC) fails in estimating azimuth DOA of the near-field source. However, the proposed algorithm performs well in localizing both near-field and far-field sources.

Two far-field sources are localized at $\left\{\theta_{1}=10^{\circ}, r_{1}=+\infty\right\}$ (i.e. $\left.\left\{\gamma_{1}=-0.2728, \phi_{1}=0\right\}\right)$ and $\left\{\theta_{2}=20^{\circ}, r_{2}=+\infty\right\}$ (i.e. $\left\{\gamma_{2}=-0.5372, \phi_{2}=0\right\}$ ), respectively. When the snapshot number and SNR are fixed respectively at 400 and $10 \mathrm{~dB}$, the scatter plot of estimated $\left(\hat{\gamma}_{l}, \hat{\phi}_{l}\right)$ pairs from 500 independent trials using the proposed algorithm, the near-field source localization algorithm (ESPRIT), and the far-field source localization algorithm (MUSIC) are shown in Figs. 13-15, respectively. From these figures, it can be seen that the near-field source 
localization algorithm fails in localizing far-field sources, but the proposed algorithm performs well in estimating azimuth DOA of the two far-field source.

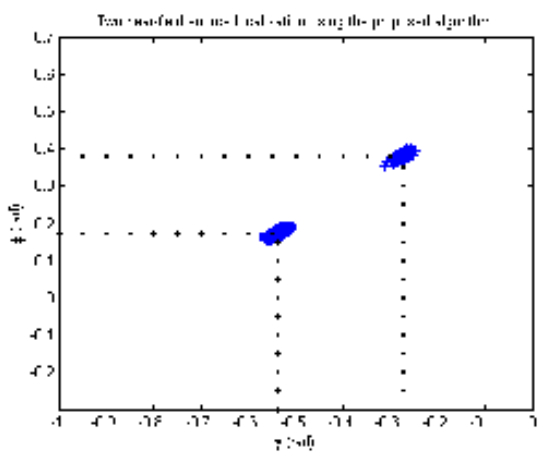

Fig. 7. Scatter plot of estimated $(\gamma, \phi)$ pairs for two near-field sources using the proposed algorithm

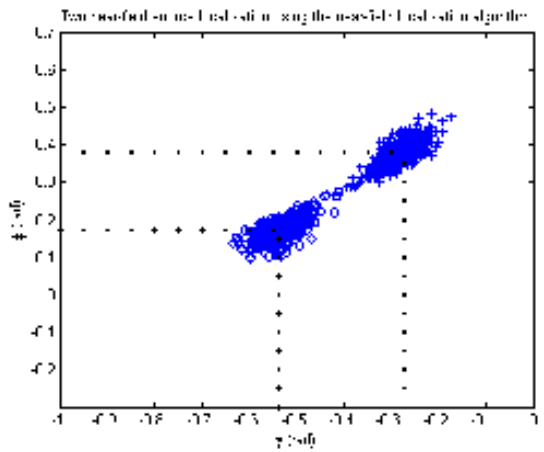

Fig. 8. Scatter plot of estimated $(\gamma, \phi)$ pairs for two near-field sources using the near-field source localization algorithm (ESPRIT)

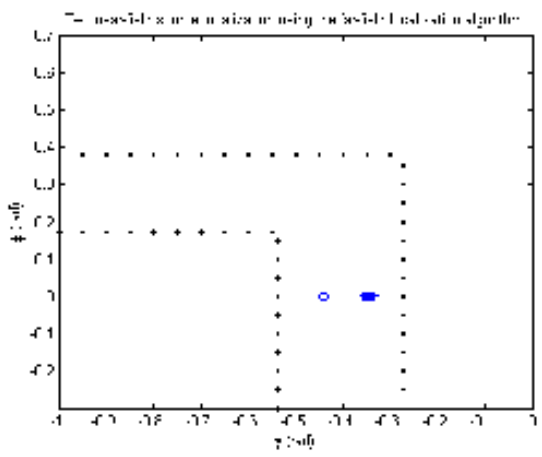

Fig. 9. Scatter plot of estimated $(\gamma, \phi)$ pairs for two near-field sources using the far-field source localization algorithm (MUSIC) 


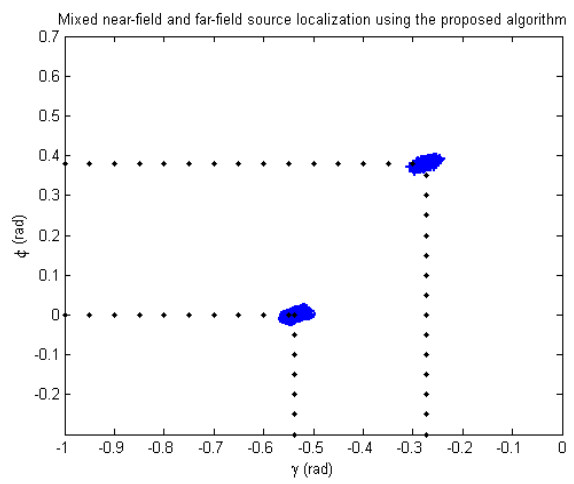

Fig. 10. Scatter plot of estimated $(\gamma, \phi)$ pairs for mixed near-field and far-field sources using the proposed algorithm

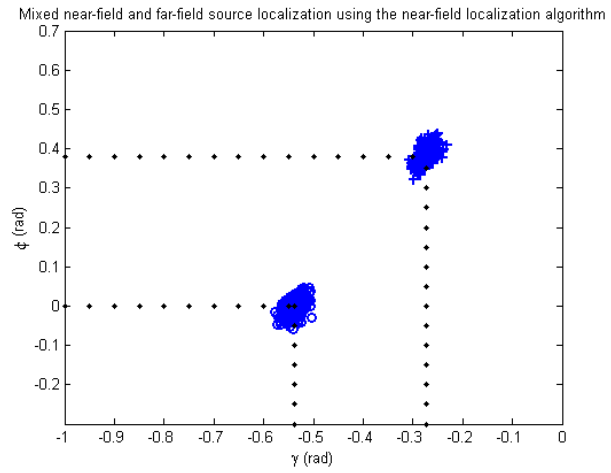

Fig. 11. Scatter plot of estimated $(\gamma, \phi)$ pairs for mixed near-field and far-field sources using the near-field source localization algorithm (ESPRIT)

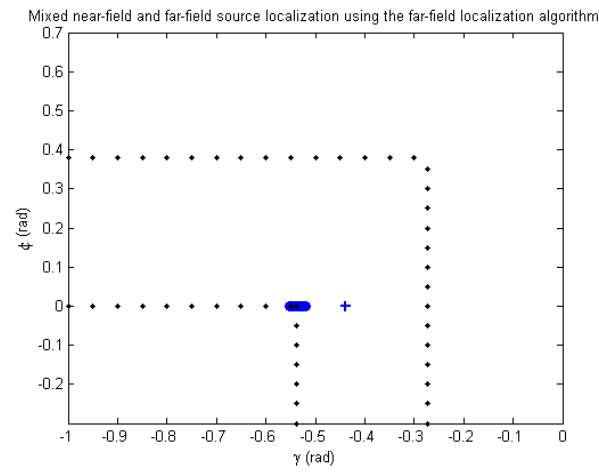

Fig. 12. Scatter plot of estimated $(\gamma, \phi)$ pairs for mixed near-field and far-field sources using the far-field source localization algorithm (MUSIC) 


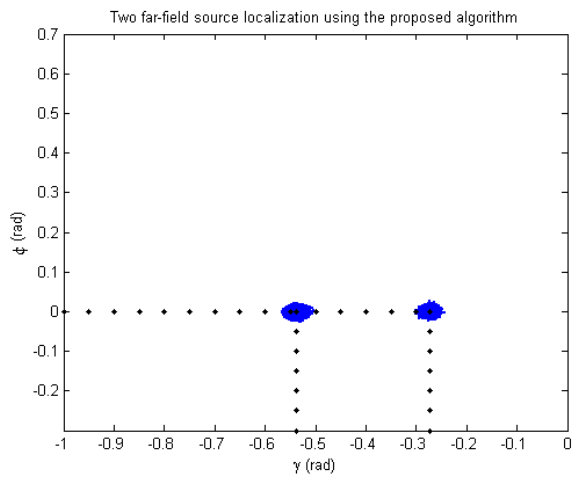

Fig. 13. Scatter plot of estimated $(\gamma, \phi)$ pairs for two far-field sources using the proposed algorithm

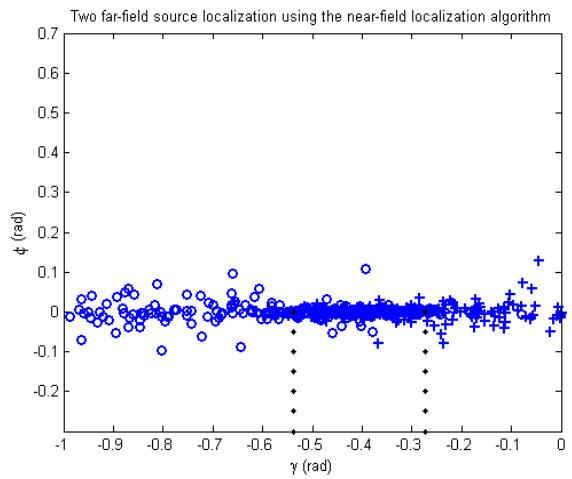

Fig. 14. Scatter plot of estimated $(\gamma, \phi)$ pairs for two far-field sources using the near-field source localization algorithm (ESPRIT)

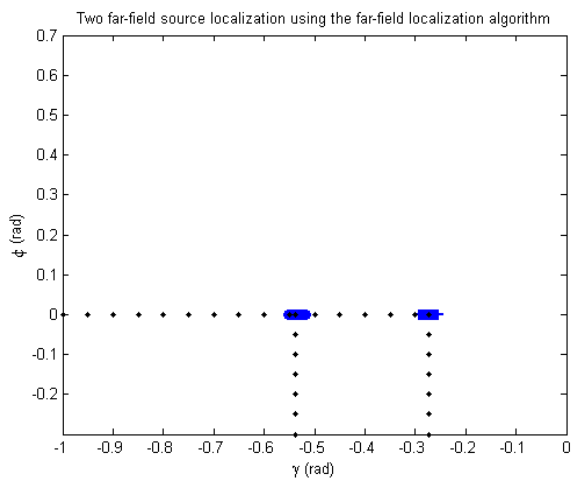

Fig. 15. Scatter plot of estimated $(\gamma, \phi)$ pairs for two far-field sources using the far-field source localization algorithm (MUSIC) 
From these simulations, it can be seen that no matter whether the received signals are nearfield sources, far-field sources or their mixture, the proposed algorithm can perform better in localizing these sources.

\section{New estimator for elevation angle}

\subsection{Proposed estimator}

When elevation angles are between 70 and 90 degrees, the estimator

$$
\operatorname{asin}\left(\frac{\lambda}{2 \pi d} \sqrt{\hat{\gamma}_{l}^{2}+\hat{\phi}_{l}^{2}}\right)
$$

may fail because imperfect estimations of $\left(\hat{\gamma}_{l}, \hat{\phi}_{l}\right)$ result in

$$
\frac{\lambda}{2 \pi d} \sqrt{\hat{\gamma}_{l}^{2}+\hat{\phi}_{l}^{2}}
$$

being greater than 1 , causing the calculation of

$$
\operatorname{asin}\left(\frac{\lambda}{2 \pi d} \sqrt{\hat{\gamma}_{l}^{2}+\hat{\phi}_{l}^{2}}\right)
$$

to fail, where $\gamma_{l}=-2 \pi d \sin \alpha_{l} \cos \beta_{l} / \lambda$ and $\phi_{l}=-2 \pi d \sin \alpha_{l} \sin \beta_{l} / \lambda$. On the other hand, some algorithms adopt another estimator

$$
\operatorname{acos}\left(\frac{-\lambda \hat{\vartheta}_{l}}{2 \pi d}\right)
$$

which is of low estimation accuracy when elevation angles are between 0 and 20 degrees $\left(\vartheta_{l}=-2 \pi d \cos \alpha_{l} / \lambda\right)$. Note that $\cos \left(20^{\circ}\right)=\sin \left(70^{\circ}\right)>0.9$ and thus it is impossible that both $\cos (\alpha)$ and $\sin (\alpha)$ are greater than 0.9 simultaneously. Therefore, $\mathrm{e}$

$$
\operatorname{asin}\left(\frac{\lambda}{2 \pi d} \sqrt{\hat{\gamma}_{l}^{2}+\hat{\phi}_{l}^{2}}\right) \text { and } \operatorname{acos}\left(\frac{-\lambda \hat{\vartheta}_{l}}{2 \pi d}\right)
$$

can be combined to form a new elevation angle estimator, which can efficiently avoid estimation failure and is of high estimation accuracy.

$$
\alpha_{l}=\left\{\begin{array}{cr}
\frac{1}{2}\left\{\operatorname{acos}\left(\frac{-\lambda \hat{\vartheta}_{l}}{2 \pi d}\right)+\operatorname{asin}\left(\frac{\lambda}{2 \pi d} \sqrt{\hat{\gamma}_{l}^{2}+\hat{\phi}_{l}^{2}}\right)\right\} & \text { if }\left(\left|\frac{\lambda \hat{\vartheta}_{l}}{2 \pi d}\right|<0.9\right) \text { and }\left(\left|\frac{\lambda}{2 \pi d} \sqrt{\hat{\gamma}_{l}^{2}+\hat{\phi}_{l}^{2}}\right|<0.9\right) \\
\operatorname{acos}\left(\frac{-\lambda \hat{\vartheta}_{l}}{2 \pi d}\right) & \text { if }\left(\left|\frac{\lambda \hat{\vartheta}_{l}}{2 \pi d}\right|<0.9\right) \text { and }\left(\left|\frac{\lambda}{2 \pi d} \sqrt{\hat{\gamma}_{l}^{2}+\hat{\phi}_{l}^{2}}\right| \geq 0.9\right) \\
\operatorname{asin}\left(\frac{\lambda}{2 \pi d} \sqrt{\hat{\gamma}_{l}^{2}+\hat{\phi}_{l}^{2}}\right) & \text { if }\left(\left|\frac{\lambda \hat{\vartheta}_{l}}{2 \pi d}\right| \geq 0.9\right) \text { and }\left(\left|\frac{\lambda}{2 \pi d} \sqrt{\hat{\gamma}_{l}^{2}+\hat{\phi}_{l}^{2}}\right|<0.9\right)
\end{array}\right.
$$




\subsection{Simulation results}

To access the effectiveness of the proposed elevation estimator, we consider a two L-shape arrays with 16 elements as shown in Fig. 16. These sensor locations are in unit of $d=\lambda / 2$. We consider a single source case $\left(e^{j 0.2 \pi k}\right)$ : elevation angle $\alpha$ and azimuth angle $\beta$ vary from $\left(0^{0}, 0^{0}\right)$ to $\left(90^{0}, 90^{0}\right)$ with $5^{0}$ increment. The snapshot number and the SNR are set to 200 and $10 \mathrm{~dB}$, respectively. The received signals are polluted by zero-mean additive white Gaussian noises. We use the root mean square error (RMSE) as the performance measure. All results provided are based on 500 independent runs. For each $(\alpha, \beta)$, we conduct 500 trials, and count the estimation failure times as well as the averaged performance only from successful trials (RMSE of elevation angle estimations versus different azimuth-elevation pair for this single source). Fig. 17 and 18 give the averaged performance counted from successful trials and the corresponding Failure Rates (FR, the failure times divided by 500) of the estimator

$$
\operatorname{asin}\left(\frac{\lambda}{2 \pi \mathrm{d}} \sqrt{\hat{\gamma}_{l}^{2}+\hat{\phi}_{l}^{2}}\right),
$$

respectively.

Since the estimator

$$
\operatorname{acos}\left(\frac{-\lambda \hat{\vartheta}_{l}}{2 \pi d}\right)
$$

shows no failure for all pair angles, we only show its averaged performance (RMSE of elevation angle estimations versus different azimuth-elevation pair for this single source) in Fig. 19.

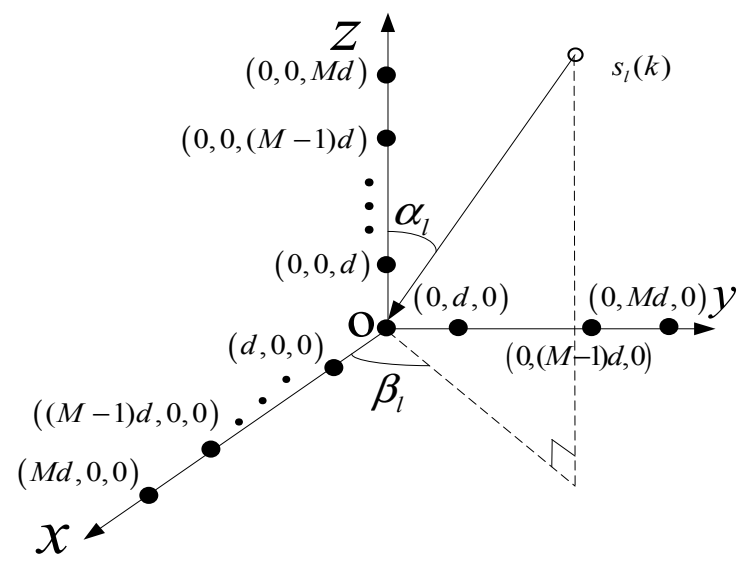

Fig. 16. Two L-shape array configuration

From Figs. 17 and 18, it can be seen that the estimator 


$$
\operatorname{asin}\left(\frac{\lambda}{2 \pi \mathrm{d}} \sqrt{\hat{\gamma}_{l}^{2}+\hat{\phi}_{l}^{2}}\right)
$$

may break downs especially when elevation angles are between $70^{\circ}$ and $90^{\circ}$. In addition, it is obvious that the estimator

$$
\operatorname{asin}\left(\frac{\lambda}{2 \pi \mathrm{d}} \sqrt{\hat{\gamma}_{l}^{2}+\hat{\phi}_{l}^{2}}\right)
$$

has lower estimation accuracy in region $\left[70^{\circ}, 90^{\circ}\right]$ than other regions. As the elevation angle approximates to $90^{\circ}$, the failure rate increases, and the related RMSE (from the successful trails) increases [15]. Although the estimator

$$
\operatorname{acos}\left(\frac{-\lambda \hat{\vartheta}_{l}}{2 \pi d}\right)
$$

shows no failure for all pair angles, we observe from Fig.19 that when the elevation angle lies in $\left[0^{0}, 20^{\circ}\right]$, the related RMSE increases. In addition, the averaged performance of the proposed estimator (RMSE of azimuth angle estimations versus different azimuth-elevation pair for this single source) is given in Fig. 20, which shows that the proposed estimator improves the performance significantly compared to

$$
\operatorname{asin}\left(\frac{\lambda}{2 \pi \mathrm{d}} \sqrt{\hat{\gamma}_{l}^{2}+\hat{\phi}_{l}^{2}}\right) \text { and } \operatorname{acos}\left(\frac{-\lambda \hat{\vartheta}_{l}}{2 \pi d}\right)
$$

especially in the regions $\left[0^{\circ}, 20^{\circ}\right]$ and $\left[70^{\circ}, 90^{0}\right]$.

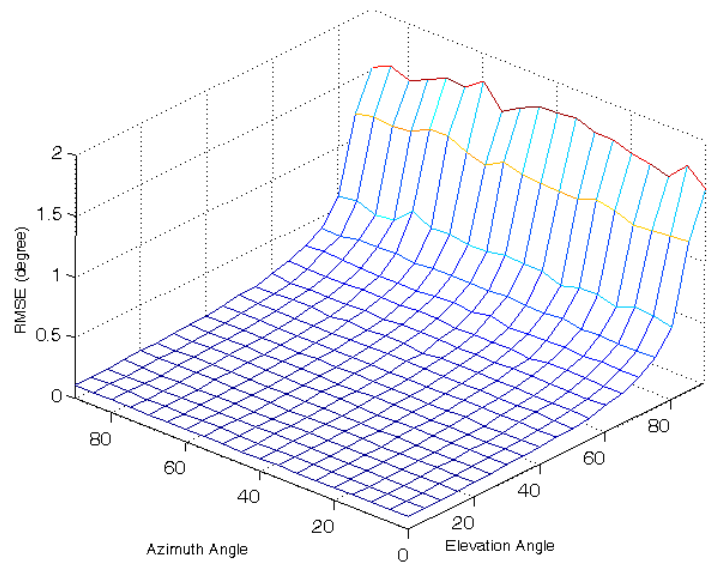

Fig. 17. Averaged performance (counted from successful trials) of elevation angle

estimations versus different azimuth-elevation pair using the estimator $\operatorname{asin}\left(\frac{\lambda}{2 \pi \mathrm{d}} \sqrt{\hat{\gamma}_{l}^{2}+\hat{\phi}_{l}^{2}}\right)$ 


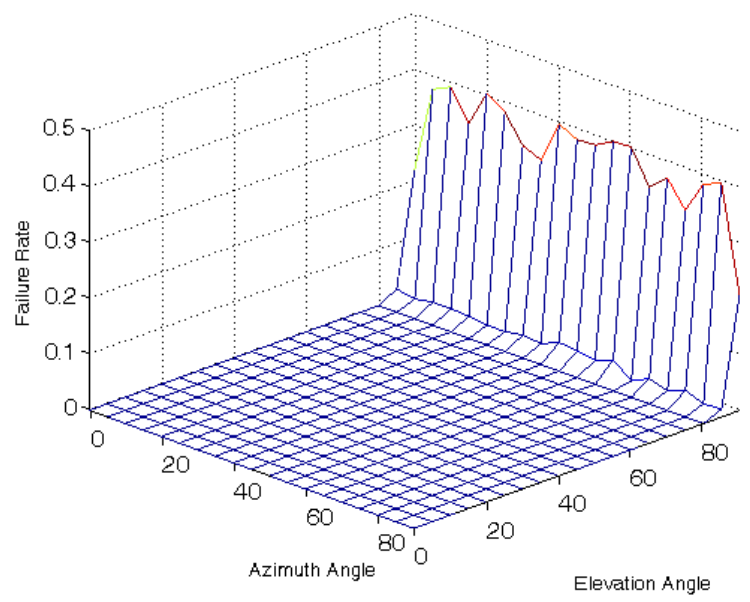

Fig. 18. Failure rates of elevation angle estimations versus different azimuth-elevation pair using the estimator $\operatorname{asin}\left(\frac{\lambda}{2 \pi \mathrm{d}} \sqrt{\hat{\gamma}_{l}^{2}+\hat{\phi}_{l}^{2}}\right)$

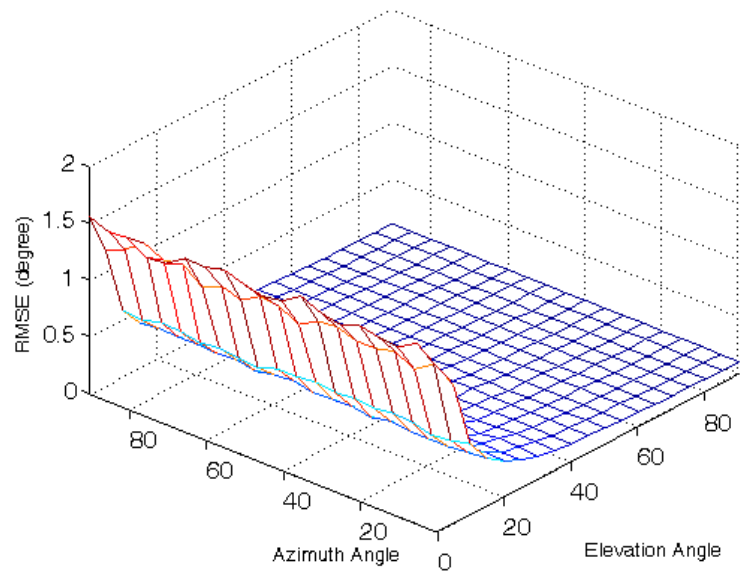

Fig. 19. Averaged performance of elevation angle estimations versus different azimuthelevation pair using the estimator $\operatorname{acos}\left(\frac{-\lambda \hat{\vartheta}_{l}}{2 \pi d}\right)$ 


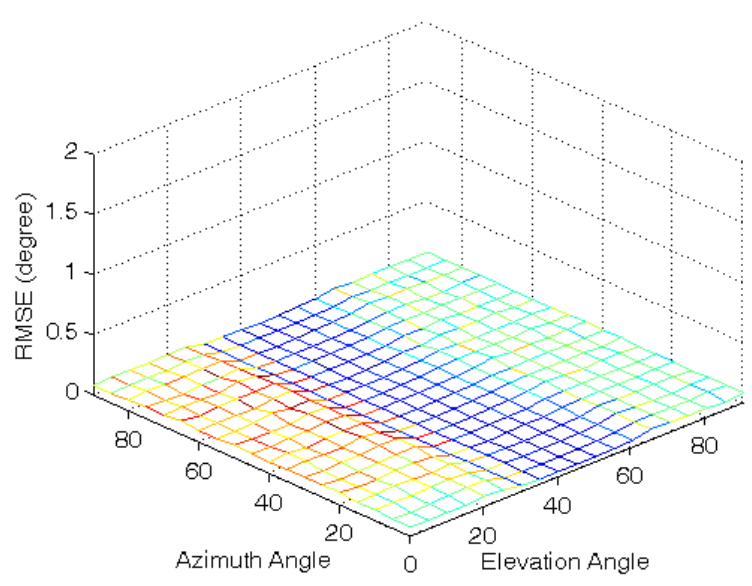

Fig. 20. Averaged performance of elevation angle estimations versus different azimuthelevation pair using the proposed estim

From these simulations, it can be seen that the proposed estimator combines

$$
\operatorname{asin}\left(\frac{\lambda}{2 \pi \mathrm{d}} \sqrt{\hat{\gamma}_{l}^{2}+\hat{\phi}_{l}^{2}}\right) \text { and } \operatorname{acos}\left(\frac{-\lambda \hat{\vartheta}_{l}}{2 \pi d}\right),
$$

and thus avoids both estimation failure and low resolution.

\section{Conclusion}

In this chapter, several novel high-resolution methods are introduced to overcome the difficulties encountered in the passive source localization of sensor array, i.e. pairing failure, mixed near-field and far-field source localization, and estimation failure problems. Although they have been developed for the uniform linear array and L-shaped array, these algorithms can be easily extended to other sensor array configurations.

\section{Acknowledgments}

This work was supported by the National Natural Science Foundation of China under Grants 61172123, 60901059 and 61075044, by China Postdoctoral Science Foundation funded project under Grant 201003679 and 20100481355, by the Natural Science Foundation under 2010JQ8001 of Shaanxi Province, and by the Discipline Union Fund under Grant 116-210905 of Xi'an University of Technology.

\section{References}

[1] Krim, H. \& Viberg M. (1996) Two Decades of Array Signal Processing Research: the Parametric Approach. IEEE Signal Processing Magazine, Vol. 13, No. 4, pp. 67-94, ISSN 1053-5888 
[2] Johnson, R. (1993) Antenna Engineering Handbook. Third Edition, New York: McGrawHill, Inc., ISBN 0-07-032381-X

[3] Roy, R. \& Kailath, T. (1989) ESPRIT-Estimation of Signal Parameters Via Rotational Invariance Techniques. IEEE Trans. Acoustics, Speech, and Signal Processing, Vol. 37, No. 7, pp. 984-995, ISSN 1053-587X

[4] Schmidt, R. (1986) Multiple Emitter Location and Signal Parameter Estimation. IEEE Trans. Antennas and Propagation, Vol. 34, No. 3, pp. 276-280, ISSN 0018-926X

[5] Marcos, S.; Marsal, A. \& Benidir, M. (1995) The Propagator Method for Source Bearing Estimation. Signal Processing, Vol. 42, No. 8, pp. 121-138, ISSN 0165-1684

[6] Hua, Y.; Sarkar, T. \& Weiner, D. (1991) An L-shaped Array for Estimation 2-D Directions of Wave Arrival. IEEE Trans. Antennas and Propagation, Vol. 39, No. 2, pp. 143-146, ISSN 0018-926X

[7] Tayem, N. \& Kwon, H. (2005) L-shape 2-dimensional Arrival Angle Estimation with Propagator Method. IEEE Trans. Antennas and Propagation, Vol. 53, No. 5, pp. 16221630, ISSN 0018-926X

[8] Harabi, F.; Gharsallah, A. \& Marcos, S.(2009) Three-dimensional Antennas Array for the Estimation of Direction of Arrival, IET Microwaves, Antennas and Propagation. Vol. 3, No. 5, pp. 843-849, ISSN 1751-8725

[9] Liang, J. \& Liu, D. (2010) Joint Elevation and Azimuth Direction Finding Using L-shaped Array. IEEE Trans. Antennas and Propagation, Vol. 58, No. 6, pp. 2136-2141, ISSN 0018-926X

[10] Pesavento, M.; Gershman, A. \& Wong, K.(2002) Direction finding in partly calibrated sensor arrays composed of multiple subarrays, IEEE Trans. Signal Processing, Vol. 50, No. 2, pp. 329-338, ISSN 1053-587X

[11] Gao, F. \& Gershman, A. (2005) A generalized ESPRIT approach to direction- of- arrival estimation, IEEE Signal Processing Letters. Vol. 12, pp. 254-257. ISSN 1070-9908

[12] Liang, J.; etc. (2011)L-shaped array-based elevation and azimuth direction finding in the presence of mutual coupling. Signal Processing, Vol. 91, No. 5, pp. 1319-1328, ISSN 0165-1684

[13] Grosicki, E.; Abed-Meraim, K. \& Hua, Y. (2005)A weighted linear prediction method for near-field source localization, IEEE Trans. Signal Processing, Vol. 53, No. 10, pp. 3651-3660, ISSN 1053-587X

[14] Yuen, N. \& Friedlander, B. (1998) Performance analysis of higher order ESPRIT for localization of near-field sources, IEEE Trans. Signal Processing, Vol. 46, No. 3, (March 1998), pp.709-719, ISSN 1053-587X

[15] Challa, R. \& Shamsunder, N. (1995) High-order subspace based algorithms for passive localization of near-field sources, in: The Twenty-Ninth Asilomar Conference on Signals, Systems \& Computers, Pacific Grove, California, USA, vol. 2, pp. 777781

[16] Liang, J. \& Liu, D. (2010) Passive localization of mixed near-field and far-field sources using two-stage MUSIC algorithm. IEEE Trans. Signal Processing, Vol. 58, No. 1, pp. 108-120, ISSN 1053-587X 
[17] Tichavsky, P.; Wong, K. \& Zoltowski, M. (2001) Near-field/far-field azimuth elevation angle estimation using a single vector-hydrophone. IEEE Trans. Signal Processing, Vol. 49, No. 11, pp. 2498-2510, ISSN 1053-587X

[18] Doclo, S. \& Moonen, M. (2003) Design of far-field and near-field broadband beamformers using eigenfilters. Signal Processing, Vol.83, No.12, pp. 2641-2673, ISSN 0165-1684

[19] Mukai, R.; Sawada, H. \& Araki, S. etc.(2006) Frequency-domain blind source separation of many speech signals using near-field and far-field models, EURASIP Journal on Applied Signal Processing, Vol. 2006, Article ID 83683, pp. 1-13, ISSN 1687-0433

[20] Dogan, M. \& Mendel, J. (1995)Applications of cumulants to array processing-Part I: aperture extension and array calibration, IEEE Trans. Signal Processing, Vol. 43, No. 5, pp. 1200-1216, ISSN 1053-587X

[21] Tayem, N. \& Kwon, H. (2006) Azimuth and elevation angle estimation with no failure and no eigen decomposition, Signal Processing, Vol. 86, No. 1, pp. 8-16, ISSN 01651684

[22] Liu, T. \& Mendel, J. (1998) Azimuth and elevation direction finding using arbitrary array geometries, IEEE Trans. Signal Processing, Vol. 46, No. 7, pp. 2061-2065, ISSN $1053-587 \mathrm{X}$

[23] Liang, J. (2009) Joint azimuth and elevation direction finding using cumulant. IEEE Sensors Journal, Vol. 9, No. 4, pp. 390-398, ISSN 1530-437X 


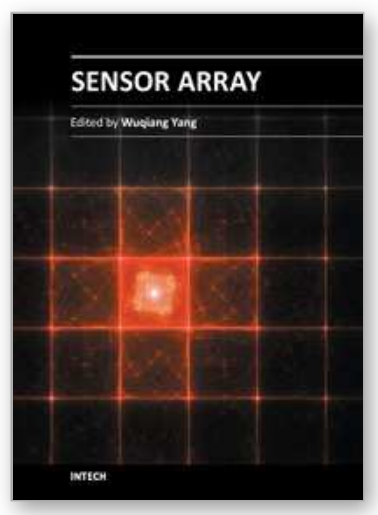

\author{
Sensor Array \\ Edited by Prof. Wuqiang Yang
}

ISBN 978-953-51-0613-5

Hard cover, 134 pages

Publisher InTech

Published online 23, May, 2012

Published in print edition May, 2012

Sensor arrays are used to overcome the limitation of simple and/or individual conventional sensors. Obviously, it is more complicated to deal with some issues related to sensor arrays, e.g. signal processing, than those conventional sensors. Some of the issues are addressed in this book, with emphasis on signal processing, calibration and some advanced applications, e.g. how to place sensors as an array for accurate measurement, how to calibrate a sensor array by experiment, how to use a sensor array to track non-stationary targets efficiently and effectively, how to use an ultrasonic sensor array for shape recognition and position measurement, how to use sensor arrays to detect chemical agents, and applications of gas sensor arrays, including e-nose. This book should be useful for those who would like to learn the recent developments in sensor arrays, in particular for engineers, academics and postgraduate students studying instrumentation and measurement.

\title{
How to reference
}

In order to correctly reference this scholarly work, feel free to copy and paste the following:

Junli Liang and Ding Liu (2012). Passive Source Localization of Sensor Arrays, Sensor Array, Prof. Wuqiang Yang (Ed.), ISBN: 978-953-51-0613-5, InTech, Available from: http://www.intechopen.com/books/sensorarray/new-progress-in-passive-source-localization-of-sensor-array

\section{INTECH}

open science | open minds

\author{
InTech Europe \\ University Campus STeP Ri \\ Slavka Krautzeka 83/A \\ 51000 Rijeka, Croatia \\ Phone: +385 (51) 770447 \\ Fax: +385 (51) 686166 \\ www.intechopen.com
}

\author{
InTech China \\ Unit 405, Office Block, Hotel Equatorial Shanghai \\ No.65, Yan An Road (West), Shanghai, 200040, China \\ 中国上海市延安西路65号上海国际贵都大饭店办公楼 405 单元 \\ Phone: +86-21-62489820 \\ Fax: $+86-21-62489821$
}


(C) 2012 The Author(s). Licensee IntechOpen. This is an open access article distributed under the terms of the Creative Commons Attribution 3.0 License, which permits unrestricted use, distribution, and reproduction in any medium, provided the original work is properly cited. 